PINHEIRO, JB; SILVA, GO; MACÊDO, AG; BISCAIA, D; RAGASSI, CF; RIBEIRO, CSC; CARVALHO, SIC; REIFSCHNEIDER, FJB. 2020. New resistance sources to root-knot nematode in Capsicum pepper. Horticultura Brasileira 38: 33-40. DOI - http://dx.doi.org/10.1590/S0102-053620200105

\title{
New resistance sources to root-knot nematode in Capsicum pepper
}

\author{
Jadir B Pinheiro ${ }^{1} \mathbb{D}$; Giovani Olegario da Silva ${ }^{2} \mathbb{D}$; Amanda G Macêdo ${ }^{3} \mathbb{D}$; Danielle Biscaia ${ }^{1} \mathbb{D}$; Carlos \\ Francisco Ragassi ${ }^{1} \mathbb{D}$; Cláudia SC Ribeiro ${ }^{1 \mathbb{D}}$; Sabrina Isabel C de Carvalho ${ }^{1} \mathbb{D}$; Francisco José B \\ Reifschneider ${ }^{1} \mathbb{D}$
}

'Embrapa Hortaliças, Brasília-DF, Brasil; jadir.pinheiro@embrapa.br; danielle.biscaia@embrapa.br; carlos.ragassi@embrapa.br; claudia. ribeiro@embrapa.br; sabrina.carvalho@embrapa.br; francisco.reifschneider@embrapa.br; ${ }^{2}$ Embrapa Hortaliças, Estação Experimental de Canoinhas, Canoinhas-SC, Brasil; giovani.olegario@embrapa.br; ${ }^{3}$ Universidade de Brasília (UnB), Brasília-DF, Brasil; amandagomesma@ gmail.com

\begin{abstract}
Thirty seven pepper genotypes, Capsicum chinense, C. annuum and $C$. frutescens, were characterized for resistance to three rootknot nematode species (Meloidogyne javanica, $M$. incognita race 1 and $M$. enterolobii) aiming to find potential resistance sources to be used in breeding programs. Three experiments were carried out, in 2013, 2014 and 2016, in a greenhouse, in randomized block design and six replicates, one plant/pot. Data were submitted to analysis of variance and average clustering. All evaluated pepper genotypes were resistant or immune to $M$. javanica. For $M$. incognita race 1, all accessions of $C$. frutescens, evaluated in the first experiment, were resistant, whereas the six accessions of $C$. chinense were susceptible. For $M$. incognita, all genotypes of $C$. chinense and $C$. annuum, evaluated in the second experiment, were resistant. In the third experiment, evaluating C. annuum genotypes, we verified that most were susceptible to $M$. incognita race 1; genotypes CNPH 30118 and CNPH 6144 were resistant, though. M. enterolobii, which is the most aggressive species, with few resistance sources described, showed a tendency of greater degree of resistance in $C$. chinense and $C$. frutescens. Although most of the evaluated genotypes were susceptible, significant differences were observed regarding the degree of susceptibility. The main contribution of this study was the identification of a genotype resistant to $M$. enterolobii, cultivar BRS Nandaia, which can be used in breeding programs.
\end{abstract}

Keywords: Capsicum spp., Meloidogyne javanica, M. incognita race $1, M$. enterolobii.

\section{RESUMO}

Novas fontes de resistência aos nematoides-das-galhas em pimenta Capsicum

Este trabalho teve como objetivo a caracterização de 37 genótipos de pimenta das espécies Capsicum chinense, $C$. annuum e $C$. frutescens, quanto à resistência a três espécies de nematoidesdas-galhas Meloidogyne javanica, M. incognita raça 1 e $M$. enterolobii, visando prospectar potenciais fontes de resistência para serem utilizadas no melhoramento. Para isso, foram realizados três experimentos em 2013, 2014 e 2016, em casa de vegetação, com delineamento em blocos casualizados com seis repetições em parcelas constituídas por uma planta/vaso. Os dados foram submetidos a análises de variância e de agrupamento de médias. Todos genótipos de pimenta avaliados foram resistentes ou imunes a $M$. javanica. Para $M$. incognita raça 1, todos genótipos de $C$. frutescens avaliados no primeiro experimento foram resistentes, enquanto que os seis genótipos de $C$. chinense foram suscetíveis. Ainda, para $M$. incognita, todos genótipos de $C$. chinense e $C$. annuum avaliados no segundo experimento, foram resistentes. No terceiro experimento, com genótipos de $C$. annuum, a maioria foi suscetível a $M$. incognita raça 1, mas os genótipos CNPH 30118 e CNPH 6144 foram resistentes. Quanto a M. enterolobii, que é a espécie mais agressiva, com poucas fontes de resistência descritas, houve uma tendência de maior grau de resistência em $C$. chinense e $C$. frutescens. Mas, apesar de a maioria dos genótipos avaliados serem suscetíveis, foram observadas diferenças significativas quanto ao grau de suscetibilidade. A principal contribuição deste trabalho foi a identificação de um genótipo resistente a $M$. enterolobii, a cultivar BRS Nandaia, sendo possível explorar esta fonte de resistência no melhoramento genético.

Palavras-chave: Capsicum spp., Meloidogyne javanica, M. incognita raça 1, M. enterolobii.

\section{Received on February 5, 2019; accepted on November 5, 2019}

$\mathrm{P}$ epper is grown in all Brazilian regions; however, no statistical analysis accurately quantifies the economic importance of this crop, since much of the production is sold in local and outdoor markets. One of the biggest problems for pepper cultivation is the root-knot nematode. These pathogens damage the plant root system and transport of water and nutrients, limiting its productivity (Kiewnick et al., 2009; Chaudhary \& Kaul, 2012).
Worldwide, the root-knot nematodes species which cause the most damages to pepper are Meloidogyne incognita, $M$. arenaria and M. javanica, found mainly in warm climates, especially in tropical and subtropical regions (Mashela \& 
Pofu, 2012; Hussain et al., 2013).

Another nematode species which has impaired vegetable production is $M$. enterolobii. This species was originally found in guava plants in 2001 in Pernambuco and Bahia States, and since then it is one of Brazilian producers' concern. In vegetables, this species was reported, for the first time, in São Paulo State, parasitizing bell pepper cv. 'Silver' rootstocks and 'Andréa' and 'Débora' tomato plants, resistant to $M$. incognita and M. javanica (Carneiro et al., 2006). Preliminary studies show that peppers of the genus Capsicum are more susceptible to $M$. enterolobii comparing to other species of root-knot nematodes (Kiewnick et al., 2009; Pinheiro et al., 2015). On the other hand, some reports on different levels of susceptibility or even resistance in pepper can be found in literature (Oliveira, 2007; Melo et al., 2011; Pinheiro et al., 2013; Gonçalves et al., 2014).

Resistance to root-knot nematodes has been associated with independent dominant genes for resistance to $M$. incognita, $M$. arenaria and $M$. javanica: N, Me1, Me2, Me3, Me4, Me5, Me6, Me7, Mech1 and Mech2. Some of these genes, such as $\mathrm{Mel}, \mathrm{Me} 3$ and $\mathrm{Me} 7$ are considered thermo-stable and effective against a wide range of Meloidogyne species, including $M$. incognita, $M$. arenaria and $M$. javanica (DjianCaporalino et al., 2011).

Due to high relevance of nematodes for pepper crop, the characterization of potential genotypes to become new cultivars or to be used for crossings to add resistance factor to other characters of agronomic importance is crucial. Therefore, this study aimed to characterize pepper genotypes in relation to resistance against three root-knot nematode species, allowing to select resistant inbred lines to be released as cultivars and/or as potential resistance sources for breeding programs.

\section{MATERIAL AND METHODS}

The experiments were carried out from April to July, 2013 - 2016, and from October to January, 2014, in Distrito Federal, Brazil, in greenhouses.
In 2013 , ten pepper genotypes were evaluated, considering six inbred lines of C. chinense 'Habanero' variety group (CNPH 15.320, CNPH 15.330, CNPH 15.332, CNPH 15.348, CNPH 15.363 and CNPH 15.367) and four inbred lines of Malagueta (C. frutescens) (CNPH 20.334, CNPH 20.699, CNPH 20.700 and CNPH 20.701). In 2014, five $C$. annuum genotypes were evaluated, being one bell pepper genotype (bell pepper cv. Tico) and four Jalapeño pepper types ('BRS Sarakura', CNPH 25.296, CNPH 25.324 and CNPH 25.313), and more six $C$. chinense sweet pepper types (CNPH 972, CNPH 3447), Habanero ('BRS Juruti', 'BRS Nandaia') and Biquinho (CNPH 35.114 and CNPH 35.122). In 2016, $16 C$. annuum genotypes belonging to variety groups Cayenne (CNPH 0029), Jalapeño (CNPH 4547, CNPH 30112, CNPH 30118, CNPH 30147, CNPH 30159, CNPH 30183, CNPH 30245) and Paprika (CNPH 6127, CNPH 6128, CNPH 6132, CNPH 6142, CNPH 6143, CNPH 6144, CNPH 6625, CNPH 6628). These were characterized in relation to $M$. javanica, $M$. incognita race 1 and M. enterolobii.

Bell pepper 'Magali' and 'Rutgers' tomato plant (Solanum lycopersicum) were used as controls in 2013, as susceptibility standards and the 'Snooker' bell pepper rootstock as resistance standard. In 2014, the same controls and tomato plants 'HF1-181' and 'Nemadoro' (resistant) and pepper genotype CNPH 3287 (C. chinense) (susceptible) were used. In 2016, 'Rutgers' and 'TH 01 ' tomato plants were used for standards of susceptibility and resistance, respectively.

The experiments were conducted in greenhouse in randomized block design in factorial scheme for each year, with six replicates, considering that the experimental unit consisted of one plant per pot with 1.5 liters sterilized substrate autoclaved at $121^{\circ} \mathrm{C}$ for 60 min. Sowings were done on April 24, 2013, October 23, 2014 and April 18, 2016. About 20 days after sowing, plants were inoculated with suspension of 5,000 eggs and eventual second stage juveniles (J2) of each isolated species, in $5 \mathrm{~mL}$ water distributed around the stem base.

At 75 days after inoculation, eggs and $\mathrm{J} 2$ were extracted from the root system of the plants according to methodology of Boneti \& Ferraz (1981). The following variables were evaluated: egg mass index (IMO): the root system was washed in running water, stained with Phloxin B solution $0.5 \mathrm{~g} / \mathrm{L}$ water for 15 minutes. Then, the authors counted the number of egg masses of nematodes using a stereoscope microscope. IMO in roots was obtained according to Taylor \& Sasser (1978), using a note scale from 0 to 5 , in which 0 is related to roots without egg masses; 1 is related to 1 to 2 egg masses; 2 corresponds to a range from 3 to 10 egg masses; 3 , from 11 to 30 egg masses; 4 , from 31-100 egg masses and 5 corresponds to more than 100 egg masses/root system. The authors also evaluated gall index (IG), which is the number of galls in each root system in each plant/replicate. IG in roots was represented by the scale from 0 to 5 , according to Taylor \& Sasser (1978), replacing the quantification of egg masses by galls and then assigning the grades. To evaluate the number of eggs per gram of roots (eggs/g), roots were washed, dried at room temperature for five hours and weighed before being processed according to Bonetti \& Ferraz (1981), counting the number of eggs of each plant divided by the weight, considering that in the experiment of 2013 this character was not evaluated. Reproduction factor (FR) was obtained dividing the final and initial population densities $(\mathrm{FR}=\mathrm{Pf} / \mathrm{Pi})$, considering zero the immune value (I), lower than 1 resistant (R) and higher than 1 susceptible (S) (Oostenbrink, 1966).

Data eggs/g roots and FR were transformed in $\sqrt{x+0.5}$ to meet the normal distribution. All data were submitted to analysis of individual and joint variance for three species of nematodes in each year, and average clustering by ScottKnott, using Genes software (Cruz, 2013).

\section{RESULTS AND DISCUSSION}

Significant interaction between 
Table 1. Evaluation of pepper accessions for resistance to root-knot nematode, 2013. Brasília, Embrapa Hortaliças, 2019.

\begin{tabular}{|c|c|c|c|c|c|}
\hline \multirow{2}{*}{ Genotypes } & \multirow{2}{*}{ Species } & \multirow{2}{*}{ Type } & IG & IMO & FR/reaction \\
\hline & & & \multicolumn{3}{|c|}{ Meloidogyne javanica } \\
\hline CNPH 15.320 & C. chinense & Habanero & $0.50 \mathrm{c}$ & $0.50 \mathrm{c}$ & $0.05 \mathrm{~b} / \mathrm{R}$ \\
\hline CNPH 15.330 & C. chinense & Habanero & $0.67 \mathrm{c}$ & $0.67 \mathrm{c}$ & $0.05 \mathrm{~b} / \mathrm{R}$ \\
\hline CNPH 15.332 & C. chinense & Habanero & $0.33 \mathrm{c}$ & $0.33 \mathrm{c}$ & $0.18 \mathrm{~b} / \mathrm{R}$ \\
\hline CNPH 15.348 & C. chinense & Habanero & $0.33 \mathrm{c}$ & $0.33 \mathrm{c}$ & $0.17 \mathrm{~b} / \mathrm{R}$ \\
\hline CNPH 15.363 & C. chinense & Habanero & $1.00 \mathrm{~b}$ & $1.00 \mathrm{~b}$ & $0.12 \mathrm{~b} / \mathrm{R}$ \\
\hline CNPH 15.367 & C. chinense & Habanero & $0.50 \mathrm{c}$ & $0.50 \mathrm{c}$ & $0.17 \mathrm{~b} / \mathrm{R}$ \\
\hline CNPH 20.334 & C. frutescens & Malagueta & $1.00 \mathrm{~b}$ & $1.00 \mathrm{~b}$ & $0.17 \mathrm{~b} / \mathrm{R}$ \\
\hline CNPH 20.699 & C. frutescens & Malagueta & $1.00 \mathrm{~b}$ & $1.00 \mathrm{~b}$ & $0.08 \mathrm{~b} / \mathrm{R}$ \\
\hline CNPH 20.700 & C. frutescens & Malagueta & $0.33 \mathrm{c}$ & $0.50 \mathrm{c}$ & $0.05 \mathrm{~b} / \mathrm{R}$ \\
\hline CNPH 20.701 & C. frutescens & Malagueta & $0.50 \mathrm{c}$ & $0.50 \mathrm{c}$ & $0.08 \mathrm{~b} / \mathrm{R}$ \\
\hline Magali & C. annuum & Bell pepper & $1.00 \mathrm{~b}$ & $1.00 \mathrm{~b}$ & $0.17 \mathrm{~b} / \mathrm{R}$ \\
\hline Snooker & C. annuиm & Bell pepper & $0.50 \mathrm{c}$ & $0.50 \mathrm{c}$ & $0.10 \mathrm{~b} / \mathrm{R}$ \\
\hline Rutgers & S. lycopersicum & Tomato & $4.50 \mathrm{a}$ & $5.00 \mathrm{a}$ & $54.38 \mathrm{a} / \mathrm{S}$ \\
\hline Average & - & - & 0.93 & 0.98 & 4.28 \\
\hline $\mathrm{CV}(\%)$ & - & - & 19.13 & 18.76 & 37.28 \\
\hline \multirow[t]{2}{*}{$\mathrm{CVg} / \mathrm{CV}$} & - & - & 1.62 & 1.75 & 3.75 \\
\hline & & & \multicolumn{3}{|c|}{ Meloidogyne incognita race 1} \\
\hline CNPH 15.320 & C. chinense & Habanero & $3.50 \mathrm{a}$ & $3.83 \mathrm{~b}$ & $7.55 \mathrm{c} / \mathrm{S}$ \\
\hline CNPH 15.330 & C. chinense & Habanero & $3.17 \mathrm{~b}$ & $4.17 \mathrm{~b}$ & $10.85 \mathrm{c} / \mathrm{S}$ \\
\hline CNPH 15.332 & C. chinense & Habanero & $3.00 \mathrm{~b}$ & $3.83 \mathrm{~b}$ & $9.28 \mathrm{c} / \mathrm{S}$ \\
\hline CNPH 15.348 & C. chinense & Habanero & $3.67 \mathrm{a}$ & $4.83 \mathrm{a}$ & $17.75 \mathrm{~b} / \mathrm{S}$ \\
\hline CNPH 15.363 & C. chinense & Habanero & $3.67 \mathrm{a}$ & $4.33 \mathrm{~b}$ & $6.18 \mathrm{c} / \mathrm{S}$ \\
\hline CNPH 15.367 & C. chinense & Habanero & $2.00 \mathrm{c}$ & $2.33 \mathrm{~d}$ & $3.20 \mathrm{~d} / \mathrm{S}$ \\
\hline CNPH 20.334 & C. frutescens & Malagueta & $1.00 \mathrm{~d}$ & $1.00 \mathrm{e}$ & $0.12 \mathrm{e} / \mathrm{R}$ \\
\hline CNPH 20.699 & C. frutescens & Malagueta & $0.83 \mathrm{~d}$ & $0.83 \mathrm{e}$ & $0.10 \mathrm{e} / \mathrm{R}$ \\
\hline CNPH 20.700 & C. frutescens & Malagueta & $1.17 \mathrm{~d}$ & $1.17 \mathrm{e}$ & $0.37 \mathrm{e} / \mathrm{R}$ \\
\hline CNPH 20.701 & C. frutescens & Malagueta & $1.00 \mathrm{~d}$ & $1.00 \mathrm{e}$ & $0.07 \mathrm{e} / \mathrm{R}$ \\
\hline Magali & C. annuum & Bell pepper & $2.83 \mathrm{~b}$ & $3.17 \mathrm{c}$ & $6.23 \mathrm{c} / \mathrm{S}$ \\
\hline Snooker & C. annuит & Bell pepper & $0.17 \mathrm{e}$ & $0.17 \mathrm{f}$ & $0.02 \mathrm{e} / \mathrm{R}$ \\
\hline Rutgers & S. lycopersicum & Tomato & $4.17 \mathrm{a}$ & $5.00 \mathrm{a}$ & $25.60 \mathrm{a} / \mathrm{S}$ \\
\hline Average & - & - & 2.32 & 2.74 & 6.71 \\
\hline CV $(\%)$ & - & - & 9.99 & 9.98 & 34.35 \\
\hline \multirow[t]{2}{*}{$\mathrm{CVg} / \mathrm{CV}$} & - & - & 2.68 & 3.04 & 1.80 \\
\hline & & & \multicolumn{3}{|c|}{ Meloidogyne enterolobii } \\
\hline CNPH 15.320 & C. chinense & Habanero & $3.00 \mathrm{~b}$ & $4.33 \mathrm{~b}$ & $2.55 \mathrm{c} / \mathrm{S}$ \\
\hline CNPH 15.330 & C. chinense & Habanero & $3.33 \mathrm{~b}$ & $4.67 \mathrm{a}$ & $4.23 \mathrm{~b} / \mathrm{S}$ \\
\hline CNPH 15.332 & C. chinense & Habanero & $3.50 \mathrm{~b}$ & $5.00 \mathrm{a}$ & $5.03 \mathrm{~b} / \mathrm{S}$ \\
\hline CNPH 15.348 & C. chinense & Habanero & $3.17 \mathrm{~b}$ & $4.17 \mathrm{~b}$ & $3.33 \mathrm{~b} / \mathrm{S}$ \\
\hline CNPH 15.363 & C. chinense & Habanero & $2.83 \mathrm{c}$ & $3.83 \mathrm{c}$ & $5.32 \mathrm{~b} / \mathrm{S}$ \\
\hline CNPH 15.367 & C. chinense & Habanero & $2.67 \mathrm{c}$ & $3.50 \mathrm{c}$ & $1.83 \mathrm{c} / \mathrm{S}$ \\
\hline CNPH 20.334 & C. frutescens & Malagueta & $3.00 \mathrm{~b}$ & $3.83 \mathrm{c}$ & $1.63 \mathrm{c} / \mathrm{S}$ \\
\hline CNPH 20.699 & C. frutescens & Malagueta & $2.67 \mathrm{c}$ & $3.17 \mathrm{c}$ & $1.95 \mathrm{c} / \mathrm{S}$ \\
\hline CNPH 20.700 & C. frutescens & Malagueta & $2.83 \mathrm{c}$ & $4.00 \mathrm{c}$ & $1.27 \mathrm{c} / \mathrm{S}$ \\
\hline CNPH 20.701 & C. frutescens & Malagueta & $2.50 \mathrm{c}$ & $3.33 \mathrm{c}$ & $1.17 \mathrm{c} / \mathrm{S}$ \\
\hline Magali & C. annuum & Bell pepper & $4.67 \mathrm{a}$ & $4.83 \mathrm{a}$ & $4.40 \mathrm{~b} / \mathrm{S}$ \\
\hline Snooker & C. annuum & Bell pepper & $2.33 \mathrm{c}$ & $4.17 \mathrm{~b}$ & $4.70 \mathrm{~b} / \mathrm{S}$ \\
\hline Rutgers & S. lycopersicum & Tomato & $5.00 \mathrm{a}$ & $5.00 \mathrm{a}$ & $34.50 \mathrm{a} / \mathrm{S}$ \\
\hline Average & - & - & 3.19 & 4.14 & 5.53 \\
\hline CV $(\%)$ & - & - & 8.31 & 6.70 & 22.32 \\
\hline $\mathrm{CVg} / \mathrm{CV}$ & - & - & 1.20 & 0.93 & 2.47 \\
\hline
\end{tabular}

IG (gall index) and IMO (egg mass) according to Taylor \& Sasser (1978) where 0) roots without egg mass and/or galls; 1) roots with 1 to 2 egg masses and/or galls; 2) roots with 3 to 10 egg masses and/or galls; 3) roots with 11 to 30 egg masses and/or galls; 4) roots with 31 to 100 egg masses and/or galls; 5) roots with over 100 egg masses and/or galls. FR/reaction (reproduction factor, final population/initial population) according to Oostenbrink (1966) where $\mathrm{I}=$ immune $(\mathrm{FR}=0) ; \mathrm{R}=$ resistant $(\mathrm{FR}<1)$ and $\mathrm{S}=$ susceptible $(\mathrm{FR}>1)$. Averages followed by same letters do not differ from each other, Scott-Knott test $(\mathrm{p}<0.05)$. CV= coefficients of environmental variation. $\mathrm{CVg} / \mathrm{CV}=\mathrm{genotypic}$ and phenotypic coefficients of variation ratio. 
genotypes and nematode species was verified in the three experiments (2013, 2014 and 2016), showing that genotype response to resistance level was different depending on the evaluated nematode species. Significant differences were noticed in all analyses of individual variance $(\mathrm{P}<0.05)$ for all characters in both experiments (data not shown).
Coefficients of environmental variation were higher for number of eggs per gram of roots (eggs/g), showing that this character is strongly influenced by environment, and that observations which were carried out using this variable would be unreliable (in the first experiment this character was not evaluated). For other characters, coefficients of variation showed lower value, and the coefficients of genotypic and environmental variation ratio $(\mathrm{CVg} / \mathrm{CV})$ were higher than the unit for most evaluated characters in the three experiments (Tables 1, 2 and 3). This result shows predominance of genetic variation, surpassing the environmental variation and, as a consequence, the

Table 2. Reaction of pepper species to root-knot nematode in 2014. Brasília, Embrapa Hortaliças, 2019.

\begin{tabular}{|c|c|c|c|c|c|c|}
\hline \multirow{2}{*}{ Genotypes } & \multirow{2}{*}{ Species } & \multirow{2}{*}{ Type } & IG & IMO & Eggs/g & FR/Reaction \\
\hline & & & \multicolumn{4}{|c|}{ M. javanica } \\
\hline Bell pepper cv. Tico & C. annuum & Bell pepper & $1.02 \mathrm{c}$ & $1.02 \mathrm{~b}$ & $13.90 \mathrm{~b}$ & $0.02 \mathrm{~b} / \mathrm{R}$ \\
\hline BRS Sarakura & C. annuиm & Jalapeño & $1.00 \mathrm{c}$ & $1.00 \mathrm{~b}$ & $3.72 \mathrm{~b}$ & $0.01 \mathrm{~b} / \mathrm{R}$ \\
\hline CNPH 25.296 & C. annuиm & Jalapeño & $1.00 \mathrm{c}$ & $1.00 \mathrm{~b}$ & $24.04 \mathrm{~b}$ & $0.04 \mathrm{~b} / \mathrm{R}$ \\
\hline CNPH 25.324 & C. annuит & Jalapeño & $1.00 \mathrm{c}$ & $1.00 \mathrm{~b}$ & $42.36 \mathrm{~b}$ & $0.06 \mathrm{~b} / \mathrm{R}$ \\
\hline CNPH 25.313 & C. annuиm & Jalapeño & $1.02 \mathrm{c}$ & $1.02 \mathrm{~b}$ & $13.64 \mathrm{~b}$ & $0.03 \mathrm{~b} / \mathrm{R}$ \\
\hline CNPH 972 & C. chinense & Sweet pepper & $1.00 \mathrm{c}$ & $1.00 \mathrm{~b}$ & $3.31 \mathrm{~b}$ & $0.01 \mathrm{~b} / \mathrm{R}$ \\
\hline CNPH 3447 & C. chinense & Sweet pepper & $1.00 \mathrm{c}$ & $1.00 \mathrm{~b}$ & $8.50 \mathrm{~b}$ & $0.04 \mathrm{~b} / \mathrm{R}$ \\
\hline BRS Juruti & C. chinense & Habanero & $1.02 \mathrm{c}$ & $1.02 \mathrm{~b}$ & $11.69 \mathrm{~b}$ & $0.05 \mathrm{~b} / \mathrm{R}$ \\
\hline BRS Nandaia & C. chinense & Habanero & $1.00 \mathrm{c}$ & $1.00 \mathrm{~b}$ & $23.06 \mathrm{~b}$ & $0.02 \mathrm{~b} / \mathrm{R}$ \\
\hline CNPH 35.114 & C. chinense & Pepper pout & $1.00 \mathrm{c}$ & $1.00 \mathrm{~b}$ & $2.88 \mathrm{~b}$ & $0.01 \mathrm{~b} / \mathrm{R}$ \\
\hline CNPH 35.122 & C. chinense & Pepper pout & $1.00 \mathrm{c}$ & $1.00 \mathrm{~b}$ & $4.52 \mathrm{~b}$ & $0.02 \mathrm{~b} / \mathrm{R}$ \\
\hline Magali & C. annuиm & Bell pepper & $1.02 \mathrm{c}$ & $1.02 \mathrm{~b}$ & $21.68 \mathrm{~b}$ & $0.06 \mathrm{~b} / \mathrm{R}$ \\
\hline Snooker & C. annuиm & Rootstock & $1.00 \mathrm{c}$ & $1.00 \mathrm{~b}$ & $22.62 \mathrm{~b}$ & $0.03 \mathrm{~b} / \mathrm{R}$ \\
\hline HF1-181 & S. lycopersicum & Tomato & $1.02 \mathrm{c}$ & $1.02 \mathrm{~b}$ & $6.86 \mathrm{~b}$ & $0.03 \mathrm{~b} / \mathrm{R}$ \\
\hline CNPH 3287 & C. chinense & Sweet pepper & $1.00 \mathrm{c}$ & $1.00 \mathrm{~b}$ & $41.40 \mathrm{~b}$ & $0.05 \mathrm{~b} / \mathrm{R}$ \\
\hline Rutgers & S. lycopersicum & Tomato & $4.98 \mathrm{a}$ & $3.83 \mathrm{a}$ & $1186.44 \mathrm{a}$ & $5.93 \mathrm{a} / \mathrm{S}$ \\
\hline Nemadoro & S. lycopersicum & Tomato & $1.33 \mathrm{~b}$ & $1.17 \mathrm{~b}$ & $7.35 \mathrm{~b}$ & $0.04 \mathrm{~b} / \mathrm{R}$ \\
\hline Average & - & - & 1.26 & 1.18 & 84.58 & 0.38 \\
\hline CV\% & - & - & 1.96 & 16.9 & 84.83 & 16.41 \\
\hline \multirow[t]{2}{*}{$\mathrm{CVg} / \mathrm{CV}$} & - & - & 4.67 & 4.03 & 1.82 & 3.74 \\
\hline & & & \multicolumn{4}{|c|}{ M. incognita race 1} \\
\hline Bell pepper cv. Tico & C. annuum & Bell pepper & $1.33 \mathrm{c}$ & $1.83 \mathrm{~b}$ & $56.83 \mathrm{~b}$ & $0.10 \mathrm{~d} / \mathrm{R}$ \\
\hline BRS Sarakura & C. annuит & Jalapeño & $1.00 \mathrm{c}$ & $1.00 \mathrm{c}$ & $26.84 \mathrm{~b}$ & $0.06 \mathrm{~d} / \mathrm{R}$ \\
\hline CNPH 25.296 & C. annuит & Jalapeño & $1.67 \mathrm{~b}$ & $1.83 \mathrm{~b}$ & $102.51 \mathrm{~b}$ & $0.15 \mathrm{~d} / \mathrm{R}$ \\
\hline CNPH 25.324 & C. annuит & Jalapeño & $2.33 \mathrm{~b}$ & $2.50 \mathrm{~b}$ & $403.55 \mathrm{~b}$ & $0.22 \mathrm{~d} / \mathrm{R}$ \\
\hline CNPH 25.313 & C. апnиит & Jalapeño & $1.50 \mathrm{c}$ & $2.17 \mathrm{~b}$ & $167.11 \mathrm{~b}$ & $0.18 \mathrm{~d} / \mathrm{R}$ \\
\hline CNPH 972 & C. chinense & Sweet pepper & $2.33 \mathrm{~b}$ & $2.11 \mathrm{~b}$ & $136.79 \mathrm{~b}$ & $0.57 \mathrm{c} / \mathrm{R}$ \\
\hline CNPH 3447 & C. chinense & Sweet pepper & $1.33 \mathrm{c}$ & $1.17 \mathrm{c}$ & $110.36 \mathrm{~b}$ & $0.39 \mathrm{c} / \mathrm{R}$ \\
\hline BRS Juruti & C. chinense & Habanero & $1.33 \mathrm{c}$ & $1.33 \mathrm{c}$ & $47.13 \mathrm{~b}$ & $0.14 \mathrm{~d} / \mathrm{R}$ \\
\hline BRS Nandaia & C. chinense & Habanero & $1.00 \mathrm{c}$ & $1.00 \mathrm{c}$ & $308.02 \mathrm{~b}$ & $0.08 \mathrm{~d} / \mathrm{R}$ \\
\hline CNPH 35.114 & C. chinense & Pepper pout & $2.00 \mathrm{~b}$ & $1.67 \mathrm{~b}$ & $175.84 \mathrm{~b}$ & $0.19 \mathrm{~d} / \mathrm{R}$ \\
\hline CNPH 35.122 & C. chinense & Pepper pout & $2.33 \mathrm{~b}$ & $2.00 \mathrm{~b}$ & $338.47 \mathrm{~b}$ & $0.56 \mathrm{c} / \mathrm{R}$ \\
\hline Magali & C. annuиm & Bell pepper & $1.00 \mathrm{c}$ & $1.00 \mathrm{c}$ & $142.83 \mathrm{~b}$ & $0.23 \mathrm{~d} / \mathrm{R}$ \\
\hline Snooker & C. апnиuт & Rootstock & $1.00 \mathrm{c}$ & $1.00 \mathrm{c}$ & $47.38 \mathrm{~b}$ & $0.07 \mathrm{~d} / \mathrm{R}$ \\
\hline HF1-181 & S. lycopersicum & Tomato & $1.00 \mathrm{c}$ & $1.00 \mathrm{c}$ & $19.79 \mathrm{~b}$ & $0.09 \mathrm{~d} / \mathrm{R}$ \\
\hline CNPH 3287 & C. chinense & Sweet pepper & $1.50 \mathrm{c}$ & $1.17 \mathrm{c}$ & $302.81 \mathrm{~b}$ & $1.18 \mathrm{~b} / \mathrm{S}$ \\
\hline Rutgers & S. lycopersicum & Tomato & $5.00 \mathrm{a}$ & $4.33 \mathrm{a}$ & $1124.24 \mathrm{a}$ & $5.18 \mathrm{a} / \mathrm{S}$ \\
\hline Nemadoro & S. lycopersicum & Tomato & $0.67 \mathrm{c}$ & $0.67 \mathrm{c}$ & $56.83 \mathrm{~b}$ & $0.13 \mathrm{~d} / \mathrm{S}$ \\
\hline Average & - & - & 1.67 & 1.63 & 207.89 & 0.57 \\
\hline CV\% & - & - & 39.12 & 37.31 & 77.78 & 22.54 \\
\hline $\mathrm{CVg} / \mathrm{CV}$ & - & - & 1.60 & 1.51 & 0.80 & 2.22 \\
\hline
\end{tabular}


Table 2 continuation

\begin{tabular}{|c|c|c|c|c|c|c|}
\hline \multirow{2}{*}{ Genotypes } & \multirow{2}{*}{ Species } & \multirow{2}{*}{ Type } & IG & IMO & Eggs/g & FR/Reaction \\
\hline & & & \multicolumn{4}{|c|}{ M. enterolobii } \\
\hline Bell pepper cv. Tico & C. annuum & Bell pepper & $5.00 \mathrm{a}$ & $4.21 \mathrm{~b}$ & $1265.77 \mathrm{~b}$ & $4.65 \mathrm{a} / \mathrm{S}$ \\
\hline BRS Sarakura & C. апnиит & Jalapeño & $4.00 \mathrm{~b}$ & $4.00 \mathrm{~b}$ & $1423.75 \mathrm{~b}$ & $4.46 \mathrm{a} / \mathrm{S}$ \\
\hline CNPH 25.296 & C. аппиит & Jalapeño & $4.00 \mathrm{~b}$ & $3.67 \mathrm{c}$ & $3279.68 \mathrm{a}$ & $6.34 \mathrm{a} / \mathrm{S}$ \\
\hline CNPH 25.324 & C. апnиит & Jalapeño & $5.00 \mathrm{a}$ & $4.20 \mathrm{~b}$ & $3906.20 \mathrm{a}$ & $6.16 \mathrm{a} / \mathrm{S}$ \\
\hline CNPH 25.313 & C. annuum & Jalapeño & $5.00 \mathrm{a}$ & $5.00 \mathrm{a}$ & $4874.49 \mathrm{a}$ & $11.42 \mathrm{a} / \mathrm{S}$ \\
\hline CNPH 972 & C. chinense & Sweet pepper & $4.67 \mathrm{a}$ & $4.17 \mathrm{~b}$ & $3231.96 \mathrm{a}$ & $8.61 \mathrm{a} / \mathrm{S}$ \\
\hline CNPH 3447 & C. chinense & Sweet pepper & $3.67 \mathrm{~b}$ & $3.67 \mathrm{c}$ & $3107.33 \mathrm{a}$ & $9.81 \mathrm{a} / \mathrm{S}$ \\
\hline BRS Juruti & C. chinense & Habanero & $3.00 \mathrm{c}$ & $3.00 \mathrm{~d}$ & $878.58 \mathrm{~b}$ & $2.44 \mathrm{~b} / \mathrm{S}$ \\
\hline BRS Nandaia & C. chinense & Habanero & $2.67 \mathrm{c}$ & $2.67 \mathrm{~d}$ & $809.31 \mathrm{~b}$ & $0.50 \mathrm{~b} / \mathrm{R}$ \\
\hline CNPH 35.114 & C. chinense & Pepper pout & $3.46 \mathrm{~b}$ & $3.46 \mathrm{c}$ & $2682.74 \mathrm{a}$ & $7.15 \mathrm{a} / \mathrm{S}$ \\
\hline CNPH 35.122 & C. chinense & Pepper pout & $4.50 \mathrm{a}$ & $4.17 \mathrm{~b}$ & $1652.94 \mathrm{a}$ & $8.53 \mathrm{a} / \mathrm{S}$ \\
\hline Magali & C. апnиит & Bell pepper & $4.83 \mathrm{a}$ & $4.50 \mathrm{~b}$ & $1065.85 \mathrm{~b}$ & $2.87 \mathrm{~b} / \mathrm{S}$ \\
\hline Snooker & C. annuum & Rootstock & $5.00 \mathrm{a}$ & $4.00 \mathrm{~d}$ & $788.27 \mathrm{~b}$ & $1.88 \mathrm{~b} / \mathrm{S}$ \\
\hline HF1-181 & S. lycopersicum & Tomato & $5.00 \mathrm{a}$ & $5.00 \mathrm{a}$ & $2143.17 \mathrm{a}$ & $10.08 \mathrm{a} / \mathrm{S}$ \\
\hline CNPH 3287 & C. chinense & Sweet pepper & $3.33 \mathrm{~b}$ & $3.33 \mathrm{c}$ & $1460.71 \mathrm{~b}$ & $5.31 \mathrm{~b} / \mathrm{S}$ \\
\hline Rutgers & S. lycopersicum & Tomato & $5.00 \mathrm{a}$ & $5.00 \mathrm{a}$ & $864.28 \mathrm{~b}$ & $7.83 \mathrm{a} / \mathrm{S}$ \\
\hline Nemadoro & S. lycopersicum & Tomato & $5.00 \mathrm{a}$ & $4.00 \mathrm{~b}$ & $334.21 \mathrm{~b}$ & $3.97 \mathrm{~b} / \mathrm{S}$ \\
\hline Average & - & - & 4.30 & 4.00 & 1986.43 & 6.00 \\
\hline $\mathrm{CV} \%$ & - & - & 14.82 & 17.48 & 53.19 & 40.89 \\
\hline $\mathrm{CVg} / \mathrm{CV}$ & - & - & 2.22 & 1.80 & 0.64 & 0.87 \\
\hline
\end{tabular}

IG (gall index) and IMO (egg mass) according to Taylor \& Sasser (1978) where 0) roots without egg mass and/or galls; 1) roots with 1 to 2 egg masses and/or galls; 2) roots with 3 to 10 egg masses and/or galls; 3) roots with 11 to 30 egg masses and/or galls; 4) roots with 31 to 100 egg masses and/or galls; 5) roots with over 100 egg masses and/or galls. Eggs/g= number of eggs per gram of roots. FR/reaction (reproduction factor, final population/initial population) according to Oostenbrink (1966) where $\mathrm{I}=\mathrm{immune}(\mathrm{FR}=0)$; $\mathrm{R}=\mathrm{resistant}(\mathrm{FR}<1)$ and $\mathrm{S}=$ susceptible $(\mathrm{FR}>1)$. Averages followed by same letters do not differ from each other, $\operatorname{Scott}-\mathrm{Knott}$ test $(\mathrm{p}<0.05)$. CV= coefficients of environmental variation. $\mathrm{CVg} / \mathrm{CV}=$ genotypic and phenotypic coefficients of variation ratio.

reliability of the estimates.

The first set of genotypes (2013) showed resistance to $M$. javanica. For $M$. incognita race $1, C$. frutescens genotypes were resistant, whereas $C$. chinense genotypes were classified as susceptible. For M. enterolobii, all evaluated genotypes, including controls used as resistant to other rootknot species, showed susceptibility. However, Habanero pepper inbred lines CNPH 15.320 and CNPH 15.367 and the set of 'Malagueta' inbred lines showed lower FR being classified as resistant (Table 1).

All genotypes belonging to the second evaluated set (2014) were resistant to $M$. javanica and $M$. incognita race 1 . For $M$. enterolobii almost all genotypes were susceptible, except pepper cultivar Habanero BRS Nandaia which showed the lowest indexes of galls (IG) and egg masses (IMO), statistically grouped with Habanero BRS Juruti, classified as resistant, with

\section{FR 0.50 (Table 2).}

For the third set of genotypes evaluated in this experiment (2016), belonging to $C$. annuum species, all showed resistance or immunity to $M$. javanica. For $M$. incognita race 1 , almost all genotypes were susceptible, except CNPH 30118 (Jalapeño type) and CNPH 6144 (Paprika type). In relation to $M$. enterolobii, all genotypes were susceptible; genotypes CNPH 0029 (Cayenne type), CNPH 4547 (Jalapeño), CNPH 30112 (Jalapeño), CNPH 6132 (Paprika) and CNPH 6144 (Paprika) showed lower FR. Genotype CNPH 6144 also showed lower values for IG and IMO (Table 3). Different reactions of these genotypes to nematodes $M$. javanica and $M$. incognita were possibly because of the specificity of some $M$ genes and resistance in $C$. annuum (Djian-Caporalino et al., 2011).

Pinheiro et al. (2013) evaluated 50 C. chinense genotypes for $M$. enterolobii and verified that all were susceptible, variation in relation to FR was verified, though, considering that 18 of these genotypes showed lower FR (1.27 to 2.83).

Similarly, Pinheiro et al. (2014) evaluated four pepper genotypes belonging to each one of $C$. chinense, C. frutescens and C. baccatum species in relation to the reaction to the same nematode species evaluated in this study and verified that $C$. chinense and $C$. baccatum genotypes were susceptible to $M$. javanica and $M$. incognita race 1, whereas $C$. frutescens genotypes were resistant. For $M$. enterolobii, all genotypes were susceptible, with lower FR for C. frutescens, in accordance with this study (Table 1).

Oliveira (2007), evaluating different Capsicum species, observed that all nine $C$. chinense genotypes were susceptible to $M$. enterolobii, with FR lower than the ones obtained in C. annuum, though. He adds that the only $C$. frutescens genotype which was evaluated was 
considered resistant, $\mathrm{FR}=0.61$. Melo et al. (2011) also verified lower reproduction indexes for $C$. chinense comparing with $C$. annuum, with higher tolerance of $C$. chinense accessions BGH-433 and BGH-4285, and also
C. annuum accessions PIM-031, PIX022I-31-07-02 and PIX-022I-31-13-01, $\mathrm{FR}=1.20,1.00,1.00,1.20$ and 0.70 , respectively.

In a similar study, Gonçalves et al. (2014) evaluated 12 C. annuum accessions, $11 C$. chinense, $10 C$. baccatum, and $6 C$. frutescens for resistance to $M$. enterolobii. FR averages for the studied species showed the following sequence: annuum (17.95) $>$ C. baccatum $(14.19)>$ C. chinense

Table 3. Reaction of pepper species to root-knot nematode in 2016. Brasília, Embrapa Hortaliças, 2019.

\begin{tabular}{|c|c|c|c|c|c|c|}
\hline \multirow{2}{*}{ Genotypes } & \multirow{2}{*}{ Species } & \multirow{2}{*}{ Type } & IG & IMO & Eggs/g & FR/reaction \\
\hline & & & \multicolumn{4}{|c|}{ M. javanica } \\
\hline CNPH 0029 & C. annuит & Cayenne & $1.00 \mathrm{~b}$ & $1.00 \mathrm{~b}$ & $34.48 \mathrm{~b}$ & $0.01 \mathrm{~b} / \mathrm{R}$ \\
\hline CNPH 4547 & C. апnиит & Jalapeño & $1.00 \mathrm{~b}$ & $1.00 \mathrm{~b}$ & $105.26 \mathrm{~b}$ & $0.06 \mathrm{~b} / \mathrm{R}$ \\
\hline CNPH 30112 & C. аппиит & Jalapeño & $1.00 \mathrm{~b}$ & $1.00 \mathrm{~b}$ & $82.46 \mathrm{~b}$ & $0.03 \mathrm{~b} / \mathrm{R}$ \\
\hline CNPH 30118 & C. аппиит & Jalapeño & $1.00 \mathrm{~b}$ & $1.00 \mathrm{~b}$ & $0.00 \mathrm{~b}$ & $0.00 \mathrm{~b} / \mathrm{I}$ \\
\hline CNPH 30147 & C. аппиит & Jalapeño & $1.00 \mathrm{~b}$ & $1.00 \mathrm{~b}$ & $9.11 \mathrm{~b}$ & $0.01 \mathrm{~b} / \mathrm{R}$ \\
\hline CNPH 30159 & C. аппиит & Jalapeño & $1.00 \mathrm{~b}$ & $1.00 \mathrm{~b}$ & $0.00 \mathrm{~b}$ & $0.00 \mathrm{~b} / \mathrm{I}$ \\
\hline CNPH 30183 & C. annuиm & Jalapeño & $1.00 \mathrm{~b}$ & $1.00 \mathrm{~b}$ & $58.25 \mathrm{~b}$ & $0.06 \mathrm{~b} / \mathrm{R}$ \\
\hline CNPH 30245 & C. аппиит & Jalapeño & $1.00 \mathrm{~b}$ & $1.00 \mathrm{~b}$ & $0.00 \mathrm{~b}$ & $0.00 \mathrm{~b} / \mathrm{I}$ \\
\hline CNPH 6127 & C. аппиит & Paprika & $1.00 \mathrm{~b}$ & $1.00 \mathrm{~b}$ & $4.45 \mathrm{~b}$ & $0.01 \mathrm{~b} / \mathrm{R}$ \\
\hline CNPH 6128 & C. аппиит & Paprika & $1.00 \mathrm{~b}$ & $1.00 \mathrm{~b}$ & $8.70 \mathrm{~b}$ & $0.01 \mathrm{~b} / \mathrm{R}$ \\
\hline CNPH 6132 & C. anпиит & Paprika & $1.00 \mathrm{~b}$ & $1.00 \mathrm{~b}$ & $19.79 \mathrm{~b}$ & $0.04 \mathrm{~b} / \mathrm{R}$ \\
\hline CNPH 6142 & C. аппиит & Paprika & $1.00 \mathrm{~b}$ & $1.00 \mathrm{~b}$ & $27.14 \mathrm{~b}$ & $0.04 \mathrm{~b} / \mathrm{R}$ \\
\hline CNPH 6143 & C. anпиит & Paprika & $1.00 \mathrm{~b}$ & $1.00 \mathrm{~b}$ & $103.70 \mathrm{~b}$ & $0.02 \mathrm{~b} / \mathrm{R}$ \\
\hline CNPH 6144 & C. аппиит & Paprika & $1.00 \mathrm{~b}$ & $1.00 \mathrm{~b}$ & $40.06 \mathrm{~b}$ & $0.07 \mathrm{~b} / \mathrm{R}$ \\
\hline CNPH 6625 & C. апnиит & Paprika & $1.00 \mathrm{~b}$ & $1.00 \mathrm{~b}$ & $29.18 \mathrm{~b}$ & $0.04 \mathrm{~b} / \mathrm{R}$ \\
\hline CNPH 6628 & C. аппиит & Paprika & $1.00 \mathrm{~b}$ & $1.00 \mathrm{~b}$ & $19.86 \mathrm{~b}$ & $0.03 \mathrm{~b} / \mathrm{R}$ \\
\hline TH 01 & S. lycopersicum & Tomato & $1.00 \mathrm{~b}$ & $1.00 \mathrm{~b}$ & $0.00 \mathrm{~b}$ & $0.00 \mathrm{~b} / \mathrm{I}$ \\
\hline Rutgers & S. lycopersicum & Tomato & $4.56 \mathrm{a}$ & $5.00 \mathrm{a}$ & $9857.79 \mathrm{a}$ & $17.73 \mathrm{a} / \mathrm{S}$ \\
\hline Average & - & - & 1.20 & 1.22 & 577.79 & 1.01 \\
\hline CV\% & - & - & 9.88 & 0.22 & 51.64 & 27.44 \\
\hline \multirow[t]{2}{*}{$\mathrm{CVg} / \mathrm{CV}$} & - & - & 7.07 & 3.54 & 5.08 & 3.19 \\
\hline & & & \multicolumn{4}{|c|}{ M. incognita race 1} \\
\hline CNPH 0029 & C. annuиm & Cayenne & $3.50 \mathrm{~b}$ & $3.50 \mathrm{~b}$ & $21781.24 \mathrm{a}$ & $12.43 \mathrm{~b} / \mathrm{S}$ \\
\hline CNPH 4547 & C. аппиит & Jalapeño & $2.67 \mathrm{c}$ & $2.67 \mathrm{c}$ & $2027.35 \mathrm{~d}$ & $2.58 \mathrm{~d} / \mathrm{S}$ \\
\hline CNPH 30112 & C. аппиит & Jalapeño & $4.50 \mathrm{a}$ & $4.50 \mathrm{a}$ & $1267.59 \mathrm{~d}$ & $5.76 \mathrm{c} / \mathrm{S}$ \\
\hline CNPH 30118 & C. аппиит & Jalapeño & $1.00 \mathrm{~d}$ & $1.00 \mathrm{~d}$ & $40.32 \mathrm{f}$ & $0.06 \mathrm{e} / \mathrm{R}$ \\
\hline CNPH 30147 & C. annuит & Jalapeño & $1.50 \mathrm{~d}$ & $1.50 \mathrm{~d}$ & $1556.29 \mathrm{e}$ & $3.27 \mathrm{~d} / \mathrm{S}$ \\
\hline CNPH 30159 & C. annuиm & Jalapeño & $2.00 \mathrm{c}$ & $2.00 \mathrm{c}$ & $736.71 \mathrm{e}$ & $2.08 \mathrm{~d} / \mathrm{S}$ \\
\hline CNPH 30183 & C. annuиm & Jalapeño & $3.50 \mathrm{~b}$ & $3.67 \mathrm{~b}$ & $3598.34 \mathrm{c}$ & $7.22 \mathrm{c} / \mathrm{S}$ \\
\hline CNPH 30245 & C. апnиит & Jalapeño & $1.67 \mathrm{~d}$ & $1.83 \mathrm{c}$ & $544.83 \mathrm{e}$ & $1.23 \mathrm{~d} / \mathrm{S}$ \\
\hline CNPH 6127 & C. аппиит & Paprika & $3.50 \mathrm{~b}$ & $3.50 \mathrm{~b}$ & $2431.42 \mathrm{~d}$ & $7.28 \mathrm{c} / \mathrm{S}$ \\
\hline CNPH 6128 & C. anпuит & Paprika & $2.50 \mathrm{c}$ & $2.50 \mathrm{c}$ & $1736.20 \mathrm{~d}$ & $4.40 \mathrm{~d} / \mathrm{S}$ \\
\hline CNPH 6132 & C. anпuит & Paprika & $1.00 \mathrm{~d}$ & $1.00 \mathrm{~d}$ & $1159.37 \mathrm{e}$ & $2.68 \mathrm{~d} / \mathrm{S}$ \\
\hline CNPH 6142 & C. anпиит & Paprika & $3.50 \mathrm{~b}$ & $3.50 \mathrm{~b}$ & $3391.53 \mathrm{c}$ & $9.17 \mathrm{~b} / \mathrm{S}$ \\
\hline CNPH 6143 & C. annuит & Paprika & $4.17 \mathrm{a}$ & $4.17 \mathrm{a}$ & $5309.42 \mathrm{c}$ & $10.44 \mathrm{~b} / \mathrm{S}$ \\
\hline CNPH 6144 & C. апnиит & Paprika & $1.00 \mathrm{~d}$ & $1.00 \mathrm{~d}$ & $12.74 \mathrm{f}$ & $0.07 \mathrm{e} / \mathrm{R}$ \\
\hline CNPH 6625 & C. апnиит & Paprika & $1.50 \mathrm{~d}$ & $1.50 \mathrm{~d}$ & $426.83 \mathrm{e}$ & $1.83 \mathrm{~d} / \mathrm{S}$ \\
\hline CNPH 6628 & C. аппиит & Paprika & $1.33 \mathrm{~d}$ & $1.33 \mathrm{~d}$ & $237.92 \mathrm{e}$ & $1.03 \mathrm{~d} / \mathrm{S}$ \\
\hline TH 01 & S. lycopersicum & Tomato & $1.00 \mathrm{~d}$ & $1.00 \mathrm{~d}$ & $7.68 \mathrm{f}$ & $0.04 \mathrm{e} / \mathrm{R}$ \\
\hline Rutgers & S. lycopersicum & Tomato & $4.83 \mathrm{a}$ & $5.00 \mathrm{a}$ & $12827.97 \mathrm{~b}$ & $27.28 \mathrm{a} / \mathrm{S}$ \\
\hline Average & - & - & 2.48 & 2.51 & 3282.99 & 5.49 \\
\hline CV\% & - & - & 25.12 & 25.66 & 37.64 & 29.76 \\
\hline $\mathrm{CVg} / \mathrm{CV}$ & - & - & 2.07 & 2.04 & 2.31 & 1.84 \\
\hline
\end{tabular}


Table 3 continuation

\begin{tabular}{|c|c|c|c|c|c|c|}
\hline \multirow{2}{*}{ Genotypes } & \multirow{2}{*}{ Species } & \multirow{2}{*}{ Type } & IG & IMO & Eggs/g & FR/reaction \\
\hline & & & \multicolumn{4}{|c|}{ M. enterolobii } \\
\hline CNPH 0029 & C. annuит & Cayenne & $4.33 \mathrm{~b}$ & $4.33 \mathrm{~b}$ & $17277.54 \mathrm{a}$ & $1.72 \mathrm{e} / \mathrm{S}$ \\
\hline CNPH 4547 & C. annuиm & Jalapeño & $5.00 \mathrm{a}$ & $5.00 \mathrm{a}$ & $2923.39 \mathrm{c}$ & $1.20 \mathrm{e} / \mathrm{S}$ \\
\hline CNPH 30112 & C. annuит & Jalapeño & $5.00 \mathrm{a}$ & $5.00 \mathrm{a}$ & $2247.74 \mathrm{c}$ & $2.22 \mathrm{e} / \mathrm{S}$ \\
\hline CNPH 30118 & C. annuиm & Jalapeño & $4.83 \mathrm{a}$ & $4.83 \mathrm{a}$ & $3967.14 \mathrm{c}$ & $3.83 \mathrm{~d} / \mathrm{S}$ \\
\hline CNPH 30147 & C. апnиит & Jalapeño & $5.00 \mathrm{a}$ & $5.00 \mathrm{a}$ & $11036.56 \mathrm{~b}$ & $5.61 \mathrm{~d} / \mathrm{S}$ \\
\hline CNPH 30159 & C. annuum & Jalapeño & $4.00 \mathrm{c}$ & $4.00 \mathrm{c}$ & $26258.02 \mathrm{a}$ & $10.25 \mathrm{~b} / \mathrm{S}$ \\
\hline CNPH 30183 & C. апnиuт & Jalapeño & $4.20 \mathrm{~b}$ & $4.20 \mathrm{~b}$ & $20625.70 \mathrm{a}$ & $5.54 \mathrm{~d} / \mathrm{S}$ \\
\hline CNPH 30245 & C. annuиm & Jalapeño & $5.00 \mathrm{a}$ & $5.00 \mathrm{a}$ & $9488.91 \mathrm{~b}$ & $9.08 \mathrm{~b} / \mathrm{S}$ \\
\hline CNPH 6127 & C. annuит & Paprika & $5.00 \mathrm{a}$ & $5.00 \mathrm{a}$ & $6535.46 \mathrm{~b}$ & $11.48 \mathrm{~b} / \mathrm{S}$ \\
\hline CNPH 6128 & C. annuиm & Paprika & $5.00 \mathrm{a}$ & $5.00 \mathrm{a}$ & $5809.48 \mathrm{~b}$ & $4.96 \mathrm{~d} / \mathrm{S}$ \\
\hline CNPH 6132 & C. annuит & Paprika & $5.00 \mathrm{a}$ & $5.00 \mathrm{a}$ & $1193.42 \mathrm{c}$ & $2.72 \mathrm{e} / \mathrm{S}$ \\
\hline CNPH 6142 & C. annuиm & Paprika & $5.00 \mathrm{a}$ & $5.00 \mathrm{a}$ & $3068.29 \mathrm{c}$ & $4.89 \mathrm{~d} / \mathrm{S}$ \\
\hline CNPH 6143 & C. annuит & Paprika & $5.00 \mathrm{a}$ & $5.00 \mathrm{a}$ & $4668.51 \mathrm{~b}$ & $7.02 \mathrm{c} / \mathrm{S}$ \\
\hline CNPH 6144 & C. anпuит & Paprika & $3.00 \mathrm{~d}$ & $3.00 \mathrm{~d}$ & $805.47 \mathrm{c}$ & $2.00 \mathrm{e} / \mathrm{S}$ \\
\hline CNPH 6625 & C. annuum & Paprika & $4.00 \mathrm{c}$ & $4.00 \mathrm{c}$ & $4425.00 \mathrm{~b}$ & $10.21 \mathrm{~b} / \mathrm{S}$ \\
\hline CNPH 6628 & C. annuит & Paprika & $4.00 \mathrm{c}$ & $4.00 \mathrm{c}$ & $2894.68 \mathrm{c}$ & $5.41 \mathrm{~d} / \mathrm{S}$ \\
\hline TH 01 & S. lycopersicum & Tomato & $5.00 \mathrm{a}$ & $5.00 \mathrm{a}$ & $5426.29 \mathrm{~b}$ & 16.88 a / S \\
\hline Rutgers & S. lycopersicum & Tomato & $5.00 \mathrm{a}$ & $4.83 \mathrm{a}$ & $7773.71 \mathrm{~b}$ & $11.20 \mathrm{~b} / \mathrm{S}$ \\
\hline Average & - & - & 4.63 & 4.62 & 7579.18 & 6.46 \\
\hline $\mathrm{CV} \%$ & - & - & 3.99 & 4.49 & 41.45 & 16.71 \\
\hline $\mathrm{CVg} / \mathrm{CV}$ & - & - & 3.10 & 2.72 & 0.97 & 1.88 \\
\hline
\end{tabular}

IG (gall index) and IMO (egg mass) according to Taylor \& Sasser (1978) where 0) roots without egg mass and/or galls; 1) roots with 1 to 2 egg masses and/or galls; 2) roots with 3 to 10 egg masses and/or galls; 3) roots with 11 to 30 egg masses and/or galls; 4) roots with 31 to 100 egg masses and/or galls; 5) roots with over 100 egg masses and/or galls. Eggs/g= number of eggs per gram of roots. FR/reaction (reproduction factor, final population/initial population) according to Oostenbrink (1966) where $\mathrm{I}=\mathrm{immune}(\mathrm{FR}=0)$; $\mathrm{R}=\mathrm{resistant}(\mathrm{FR}<1)$ and $\mathrm{S}=$ susceptible $(\mathrm{FR}>1)$. Averages followed by same letters do not differ from each other, $\operatorname{Scott}-\operatorname{Knott}$ test $(\mathrm{p}<0.05)$. CV= coefficients of environmental variation. $\mathrm{CVg} / \mathrm{CV}=$ genotypic and phenotypic coefficients of variation ratio.

(12.30) > C. frutescens (9.26), considering the $C$. chinense accession UENF $1730(\mathrm{FR}=0.30)$ resistant to $M$. enterolobii.

All pepper genotypes evaluated in this study were resistant or immune to $M$. javanica. Four $C$. frutescens genotypes evaluated in the first experiment were resistant to $M$. incognita, whereas six $C$. chinense genotypes, in the same experiment, were susceptible. For $M$. incognita race 1 , all $C$. chinense and C. annuum genotypes evaluated in the second experiment were resistant. In the third experiment, in which only C. annuum genotypes were evaluated, most were susceptible to $M$. incognita, but genotypes CNPH 30118 and CNPH 6144 were resistant. In relation to $M$. enterolobii, which is the most aggressive species, and few sources of resistance described in literature, a tendency of higher degree of resistance in $C$. chinense and $C$. frutescens was observed and, although most of the genotypes evaluated in the present study were susceptible, significant differences were observed regarding the degree of susceptibility. This information on different levels of resistance to root-knot nematodes among and in Capsicum species is of extreme importance for breeding programs, yet the main contribution of this study was the identification of resistance to M. enterolobii in 'BRS Nandaia', a $C$. chinense genotype. Nevertheless, further studies are necessary to investigate molecular bases of this resistance.

\section{REFERENCES}

BONETI, JIS; FERRAZ, S. 1981. Modificações do método de Hussey \& Barker para extração de ovos de Meloidogyne exigua em raízes de cafeeiro. Fitopatologia Brasileira 6: 553-553.

CARNEIRO, RMDG; ALMEIDA, MRA; BRAGA, RS; ALMEIDA, CA; GIORIA
R. 2006. Primeiro registro de Meloidogyne mayaguensis parasitando plantas de tomate e pimentão resistentes à meloidoginose no estado de São Paulo. Nematologia Brasileira 30: 81-86.

CHAUDHARY, KK; KAUL, RK. 2012. Compatibility of Pausteria penetrans with fungal parasite Paecilomyces lilacinus against root knot nematode on chilli: Capsicum annuum. South Asian Journal Experimental Biology 1: 36-42.

CRUZ, CD. 2013. Genes: a software package for analysis in experimental statistics and quantitative genetics. Acta Scientiarum Agronomy 35: 271-276.

DJIAN-CAPORALINO, C; MOLINARI, S; PALLOIX, A; CIANCIO, A; FAZARI, A; MARTEU, N; CASTAGNONE-SERENO, P. 2011. The reproductive potential of the root-knot nematode Meloidogyne incognita is affected by selection for virulence against major resistance genes from tomato and pepper. European Journal of Plant Pathology 131: 431-440.

GONÇALVES, LSA; GOMES, VM; ROBAINA, RR; VALIM， RH; RODRIGUES, R; ARANHA, FM. 2014. Resistência a Meloidogyne enterolobii em acessos de Capsicum spp. Agrária 9: 49-52. 
HUSSAIN, F; SHAUKAT, SS; ABID, M; USMAN, F; AKBAR, M. 2013. Control of Meloidogyne javanica and Fusarium solani in chilli (Capsicum annuum L.) with the application of chitin. Pakistan Journal of Nematology 31: 165-170.

KIEWNICK, S; DESSIMOZ, M; FRANCK, L. 2009. Effects of the Mi-1 and the N root-knot nematode-resistance gene on infection and reproduction of Meloidogyne enterolobii on tomato and pepper cultivars. Journal of Nematology 41: 134-139.

MASHELA, P; POFU, K. 2012. Host response of Capsicum frutescens cultivar 'Capistrano' to Meloidogyne incognita race 2. Acta Agriculturae Scandinavica 62: 765-768.

MELO, OD; MALUF, WR; GONÇALVES, RJS;
NETO, ACG; GOMES, LAA; CARVALHO, RC. 2011. Triagem de genótipos de hortaliças para resistência a Meloidogyne enterolobii. Pesquisa Agropecuária Brasileira 46: 829835.

OLIVEIRA, CD. 2007. Enxertia de plantas de pimentão em Capsicum spp. no manejo de nematoides de galha. Jaboticabal: UNESP. $134 \mathrm{p}$ (Ph.D. Thesis).

OOSTENBRINK, M. 1966. Major characteristics of the relation between nematodes and plants. Mededelingen Landbouw 66: 1-46.

PINHEIRO, JB; REIFSCHNEIDER, FJ; PEREIRA, RB; MOITA, AW. 2013. Reprodução de Meloidogyne enterolobii em pimentas Capsicum dos grupos Habanero e Murupi. Nematologia Brasileira 37: 61-65.
PINHEIRO, JB; REIFSCHNEIDER, FJB; PEREIRA, RB; MOITA, AW. 2014. Reação de genótipos de Capsicum ao nematoide-dasgalhas. Horticultura Brasileira 32: 371-375.

PINHEIRO, JB; BOITEUX, LS; ALMEIDA, MRA; PEREIRA, RB; GALHARDO, LC; CARNEIRO, RMDG. 2015. First report of Meloidogyne enterolobii in Capsicum rootstocks carrying the $\mathrm{Mel}$ and $\mathrm{Me} 3 / \mathrm{Me} 7$ genes in central Brazil. Nematropica 45: 184-188.

TAYLOR, A; SASSER, JN. 1978. Biology, identification and control of root-knot nematodes (Meloidogyne species). United States: North Caroline State University Graphics. 111p. 